\title{
Supporting Information for Kinetic and Microscopic Studies on Reductive Transformations of Organic Contaminants on Goethite
}

\section{Environmental Science and Technology}

\author{
Chan Lan Chun ${ }^{1}$, R. Lee Penn ${ }^{2}$, and William A. Arnold ${ }^{1}$
}

${ }^{1}$ Department of Civil Engineering, University of Minnesota, 500 Pillsbury Dr. SE, Minneapolis, Minnesota 55455-0116

${ }^{2}$ Department of Chemistry, University of Minnesota, 207 Pleasant St SE, Minneapolis, MN 55455-0431

March 15, 2006

Section S1. Additional experimental information

Page

Section S2. Kinetic modeling

Section S3. T-Test for goethite particles

Section S4. Calculation of the predicted lengths of new goethite via mass balance S5

Section S5. Visual MINTEQ input and output

Table S1. Properties of goethite nanorods

Table S2. Surface area of goethite nanoparticles after sequential spike experiments

Figure S1. Schematic diagram of goethite nanorod

Figure S2 Degradation of 4-Cl-NB and TCNM in sequential spike experiments

Figure S3. Loss of aqueous Fe(II) in sequential spike experiments

Figure S4. X-ray diffraction patterns of (a) pre- and (b) post-reaction goethite $\quad$ S9

$\begin{array}{ll}\text { Figure S5. Aqueous Fe(II) concentration in control experiments } & \text { S10 }\end{array}$

Figure S6. Amount of Fe(II) sorbed onto goethite in sequential spike experiment with 4-Cl-NB $\quad$ S12 


\section{Section S1. Additional Experimental Information}

\section{Chemicals}

The following chemicals were purchased from Sigma-Aldrich and used as received: trichloronitromethane (TCNM, 99.6\%), nitromethane (NM, 99.3\%), 1-chloro-4-nitrobenzene (4chloronitrobenzene; 4-Cl-NB, 99\%), and 4-chloroaniline (4-Cl-An, 98\%). Dichloronitromethane (DCNM; 95.13\%) and chloronitromethane (CNM; 92.89\%) were obtained from CanSyn Chemical Corporation. All aqueous solutions were prepared with ultrapure water (Milli-Q, Millipore). 3-morpholinopropanesulfonic acid (MOPS; 99.5\%, Sigma; $50 \mathrm{mM}$ ) buffer was adjusted to $\mathrm{pH} 7$ with $5 \mathrm{M} \mathrm{NaOH}(97 \%$, Aldrich) and then was deoxygenated by argon sparging. Ultra-high purity Argon was obtained from TriState Airgas, and was purified using an in-line molecular sieve and oxygen traps. For high-pressure liquid chromatography (HPLC) and gas chromatography (GC) analyses, acetonitrile (HPLC grade), potassium phosphate monobasic $(100 \%)$, potassium phosphate dibasic $(100 \%)$, and n-pentane were obtained from Fisher. $\mathrm{FeCl}_{2}$ (98\%, Aldrich), $\mathrm{FeCl}_{3} \cdot 6 \mathrm{H}_{2} \mathrm{O}\left(99 \%\right.$, Fisher), $\mathrm{NaHCO}_{3}$ (Fisher, $100.2 \%$ ) and $\mathrm{Fe}\left(\mathrm{NO}_{3}\right)_{3} \cdot 9 \mathrm{H}_{2} \mathrm{O}$ $(98.7 \%$, Fisher) were used to prepare stock solutions of Fe(II) and to synthesize goethite nanorods. 3-(2-pyridyl)-5, 6-diphenyl-1, 2, 4-triazine-4, 40-disulfonic acid monosodium salt hydrate (FerroZine reagent, 97\%, Aldrich), hydroxylamine hydrochloride (99\%, Aldrich), ammonium hydroxide (29.7\% solution, Mallinckrodt), hydrochloric acid (37.4\%, Mallinckrodt), and ammonium acetate (98\%, Mallinckrodt) were used for quantification of ferrous iron.

\section{Characterization of Goethite Nanorods}

The synthesized goethite nanoparticles were washed by placing the suspension in a dialysis bag $(\mathrm{MW}=2000)$ and the dialysis water changed many times over several days. Then, the resulting material was dried in a fume hood. The dry, deep orange particles were ground using a mortar and pestle and stored in a glass vial. XRD (Co $\mathrm{K}_{\alpha 1}(=1.789 \AA)$ radiation, Philips Xpert) confirmed that the product was goethite, and no other iron oxides were detected. The particle size of over 500 particles was measured from calibrated TEM images. Results showed that the goethite crystals were acicular with average dimensions of $63 \mathrm{~nm} \times 9 \mathrm{~nm}$ and a broad particle size distribution with aspect ratios ranging between $2: 1$ and 22:1. The (110) faces correspond to the sides of these rods and (021) faces form the ends (Pbnm space group). The lengths and widths of goethite particles are defined by $c$ - and $b$-axes, respectively (Figure $\mathrm{S} 1$ ). The properties of goethite nanorods are shown in Table $\mathrm{S} 1$.

\section{Analytical Methods}

4-Cl-NB and 4-Cl-An were analyzed using high-pressure liquid chromatography (LC module 1 plus, Waters) equipped with a $\mathrm{C}_{18}$ column (Supelco, $15 \mathrm{~cm} \times 4.6 \mathrm{~mm}, 3 \mu \mathrm{m}$ particle size). The injection volume was $10 \mu \mathrm{L}$ and the detector wavelength was $254 \mathrm{~nm}$. The mobile phase was $5 \mathrm{mM} \mathrm{pH} 7$ phosphate buffer/acetonitrile $(3 / 2 \mathrm{v} / \mathrm{v})$ at a flow rate of $1 \mathrm{~mL} / \mathrm{min}$. TCNM and its degradation products were detected and quantified via on-column injection of a $1 \mu \mathrm{L}$ sample of the n-pentane extract by an AS2000 liquid autosampler (ThermoQuest) onto a DB-1 column $(30 \mathrm{~m} \times 0.32 \mathrm{~mm}$ i.d. $\times 5 \mu \mathrm{m}$ film thickness, J\&W Scientific). The gas chromatograph was a Trace GC (ThermoQuest). The temperature of the electron capture detector was $290{ }^{\circ} \mathrm{C}$. The oven temperature was initially held at $40{ }^{\circ} \mathrm{C}$ for 5 minutes. This was followed by a 10 ${ }^{\circ} \mathrm{C} /$ minute temperature ramp to $180^{\circ} \mathrm{C}$ and then a $20^{\circ} \mathrm{C} /$ minute temperature increase to $240^{\circ} \mathrm{C}$. The temperature was then maintained at $240^{\circ} \mathrm{C}$ for 0.5 minutes. 


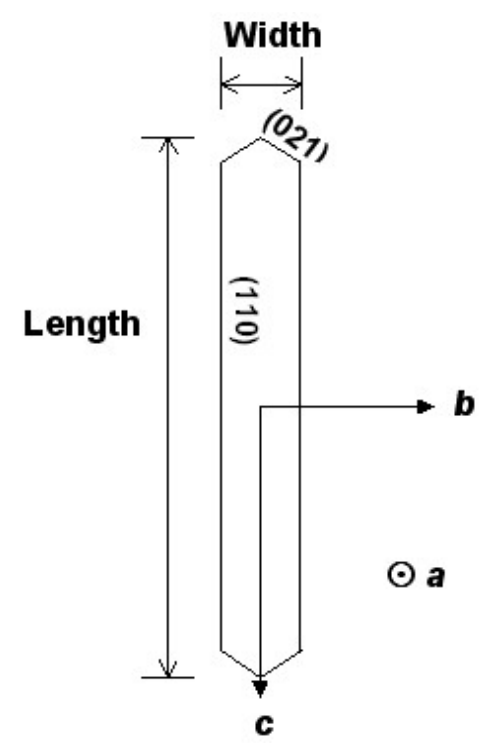

Figure S1. Schematic diagram of goethite nanorod

Table S1. Properties of goethite nanorods

\begin{tabular}{|c|c|c|c|c|}
\hline Iron Mineral & $\begin{array}{c}\mathrm{SA}_{\mathrm{BET}}{ }^{2} \\
\left(\mathrm{~m}^{2} / \mathrm{g}\right)\end{array}$ & $\begin{array}{c}\mathrm{SA}_{\mathrm{TEM}}^{2} \\
\left(\mathrm{~m}^{2} / \mathrm{g}\right)\end{array}$ & $\begin{array}{c}\text { Width }^{3} \\
(\mathrm{~nm})\end{array}$ & $\begin{array}{c}\text { Length }^{3} \\
(\mathrm{~nm})\end{array}$ \\
\hline $\begin{array}{c}\text { Goethite } \\
\text { Nanorod }\end{array}$ & $136.8 \pm 0.5$ & $218.6 \pm 86.4$ & $8.9 \pm 3.9$ & $63.7 \pm 22.9$ \\
\hline
\end{tabular}

${ }^{1}$ Surface area measure via gas absorption/desorption (BET measurement).

${ }^{2}$ Surface area calculated from TEM measurements.

${ }^{3}$ Width and length are defined in Figure S1.

Section S2. Kinetic Modeling

Reaction kinetics for organic compounds were fit using a pseudo-first-order reaction model:

$$
\frac{\mathrm{dC}}{\mathrm{dt}}=-\mathrm{k}_{4-\mathrm{Cl}-\mathrm{NB} \text { or TCNM }} \mathrm{C}
$$

where $\mathrm{C}=$ concentration of $4-\mathrm{Cl}-\mathrm{NB}$ or TCNM

$\mathrm{k}_{4-\mathrm{Cl}-\mathrm{NB} \text { or } \mathrm{TCNM}}=$ pseudo-first-order rate constant of 4-Cl-NB or TCNM.

Loss of aqueous $\mathrm{Fe}(\mathrm{II})$ were fit using pseudo-zero-order and pseudo-first-order reaction model:

$$
\begin{aligned}
& \frac{\mathrm{dFe}}{\mathrm{dt}}=-\mathrm{k}_{\mathrm{Fe}(\mathrm{II})_{\mathrm{aq}}}^{0} \\
& \frac{\mathrm{dFe}}{\mathrm{dt}}=-\mathrm{k}_{\mathrm{Fe}(\mathrm{II})_{\mathrm{aq}}} \mathrm{Fe}
\end{aligned}
$$

where $\mathrm{Fe}=$ concentration of aqueous $\mathrm{Fe}(\mathrm{II})$

$\mathrm{k}_{\mathrm{Fe}(\mathrm{II})_{\mathrm{aq}}}^{0}=$ pseudo-zero-order rate constant of loss of aqueous $\mathrm{Fe}(\mathrm{II})$

$\mathrm{k}_{\mathrm{Fe}(\mathrm{II})_{\mathrm{aq}}}=$ pseudo-first-order rate constant of loss of aqueous $\mathrm{Fe}(\mathrm{II})$. 

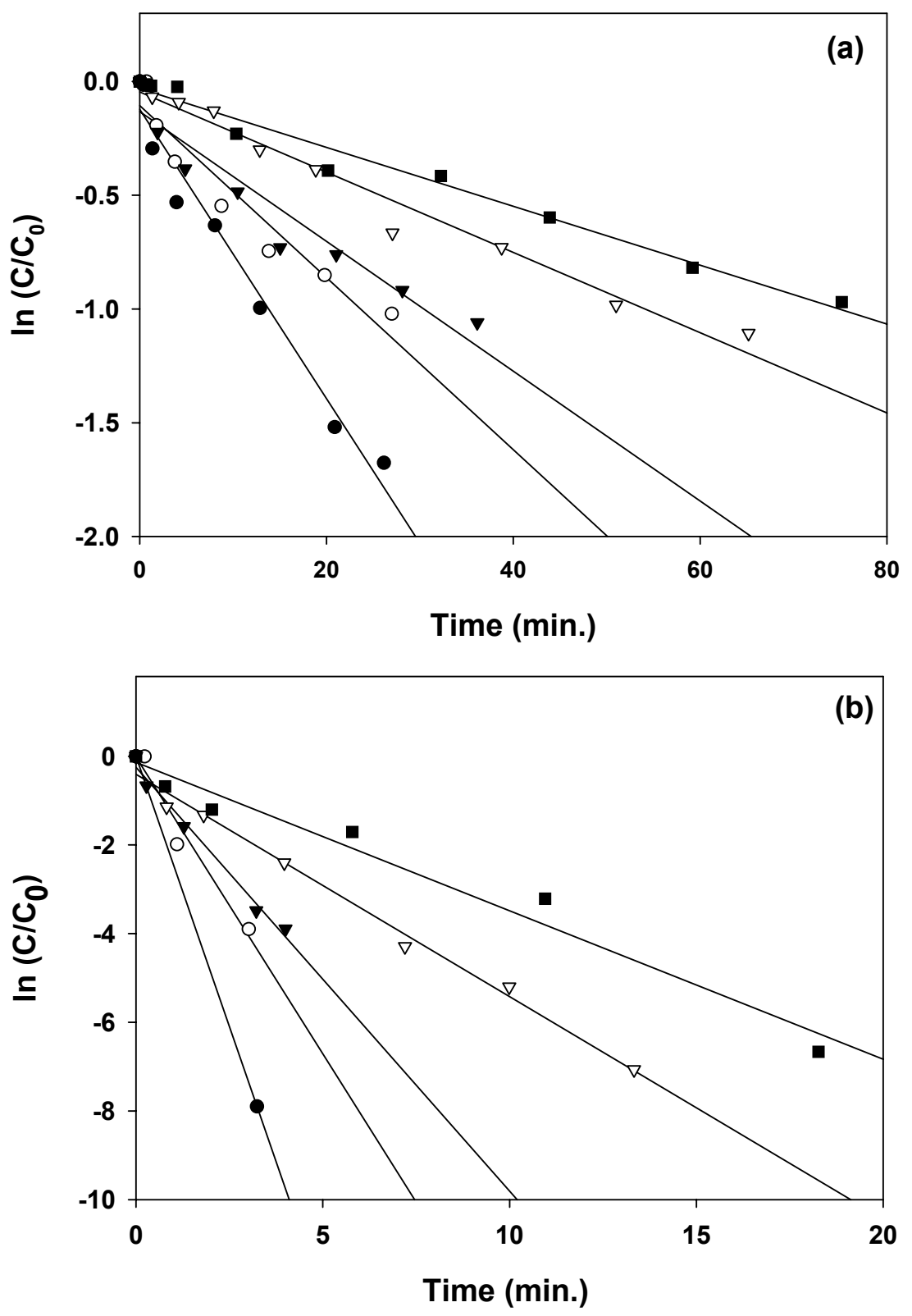

Figure S2. Degradation of 4-Cl-NB (a) and TCNM (b) in sequential spike experiments. Experiments were initiated by spiking the organic compounds into a Fe(II)-goethite suspension to achieve an initial concentration of $100 \mu \mathrm{M}$. Data points represent the following: $1^{\text {st }}$ injection, $\boldsymbol{O} ; 2^{\text {nd }}$ injection, $\bigcirc ; 3^{\text {rd }}$ injection, $\nabla ; 4^{\text {th }}$ injection, $\nabla ; 5^{\text {th }}$ injection, $\boldsymbol{\square}$. Solid lines are the linear regressions. 

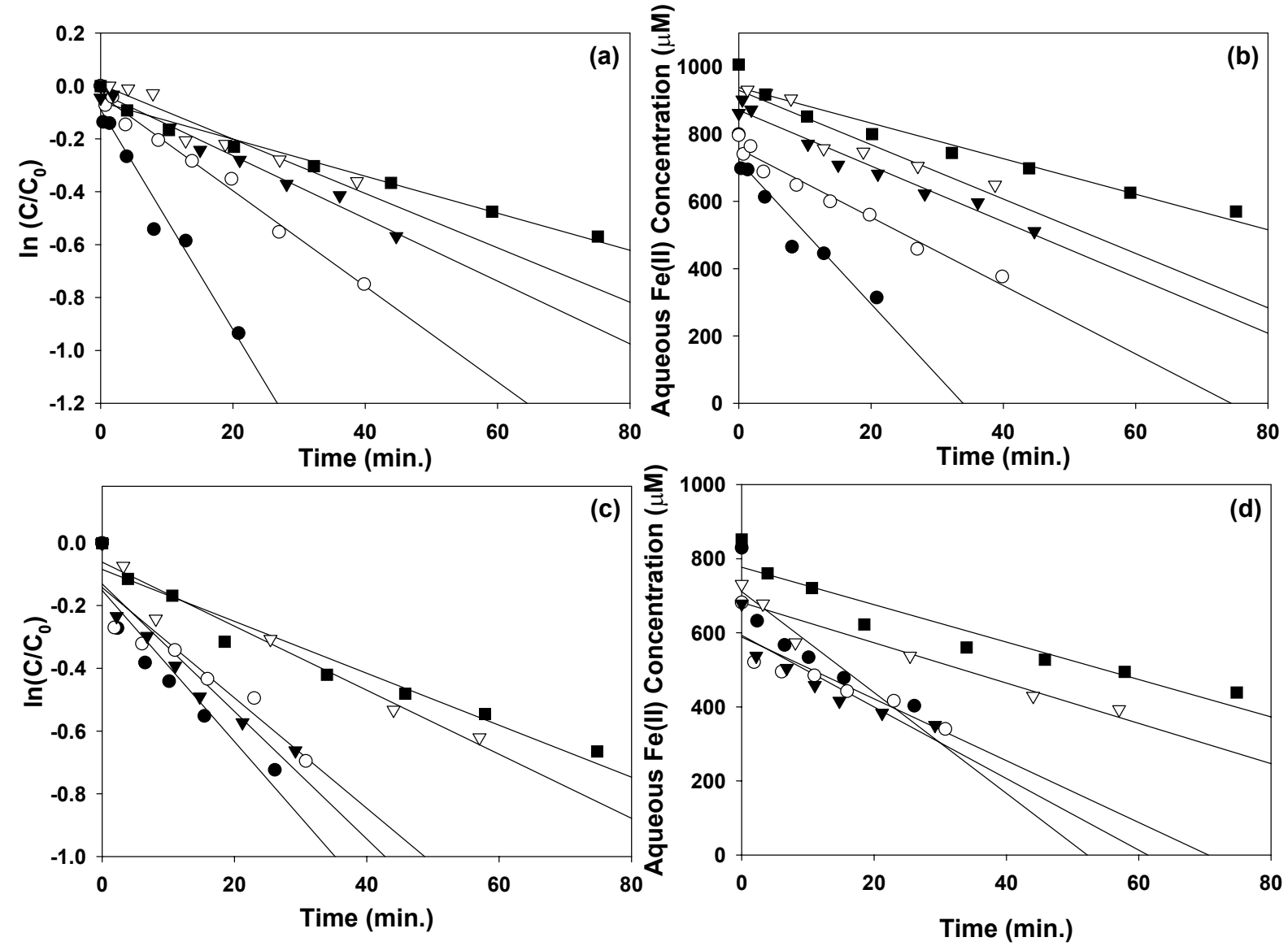

Figure S3. Loss of aqueous Fe(II) in the degradation of 4-Cl-NB (a, b) and TCNM (c, d) in sequential spike experiments. Data points represent the following: $1^{\text {st }}$ injection, $\mathbf{0} ; 2^{\text {nd }}$ injection, $\bigcirc ; 3^{\text {rd }}$ injection, $\boldsymbol{\nabla} ; 4^{\text {th }}$ injection, $\nabla ; 5^{\text {th }}$ injection, $\mathbf{\square}$. Solid lines are linear regressions. 


\section{Section S3. T-test for goethite particles}

t-test: Two-Sample Assuming Unequal Variances (Alpha level: 0.05)

1. Length

(a) 4-Cl-NB

\begin{tabular}{|c|c|c|}
\hline & $1^{\text {st }}$ Injection & Before \\
\hline Mean & 71.1 & 63.7 \\
\hline Variance & 654 & 527 \\
\hline Observations & 505 & 663 \\
\hline Hypothesized Mean Difference & 0 & \\
\hline df & 1019 & \\
\hline t Stat & 5.13 & \\
\hline $\mathrm{P}(\mathrm{T}<=\mathrm{t})$ one-tail & 1.7E-07 & \\
\hline t Critical one-tail & 1.65 & \\
\hline $\mathrm{P}(\mathrm{T}<=\mathrm{t})$ two-tail & 3.4E-07 & \\
\hline \multirow[t]{2}{*}{ t Critical two-tail } & 1.96 & \\
\hline & $2^{\text {nd }}$ Injection & $1^{\text {st }}$ Injection \\
\hline Mean & 75.3 & 71.1 \\
\hline Variance & 656 & 654 \\
\hline Observations & 419 & 505 \\
\hline Hypothesized Mean Difference & 0 & \\
\hline df & 890 & \\
\hline t Stat & 2.45 & \\
\hline $\mathrm{P}(\mathrm{T}<=\mathrm{t})$ one-tail & 7.3E-03 & \\
\hline t Critical one-tail & 1.65 & \\
\hline$P(T<=t)$ two-tail & $1.5 \mathrm{e}-02$ & \\
\hline \multirow[t]{2}{*}{ t Critical two-tail } & 1.96 & \\
\hline & $3^{\text {rd }}$ Injection & $2^{\text {nd }}$ Injection \\
\hline Mean & 85.1 & 75.3 \\
\hline Variance & 756 & 656 \\
\hline Observations & 402 & 419 \\
\hline Hypothesized Mean Difference & 0 & \\
\hline$d f$ & 809 & \\
\hline t Stat & 5.30 & \\
\hline$P(T<=t)$ one-tail & 7.38E-08 & \\
\hline t Critical one-tail & 1.65 & \\
\hline$P(T<=t)$ two-tail & $1.48 \mathrm{E}-07$ & \\
\hline t Critical two-tail & 1.96 & \\
\hline
\end{tabular}




\begin{tabular}{|c|c|c|}
\hline & $4^{\text {th }}$ Injection & $3^{\text {rd }}$ Injection \\
\hline Mean & 92.2 & 85.1 \\
\hline Variance & 933 & 756 \\
\hline Observations & 502 & 402 \\
\hline Hypothesized Mean Difference & 0 & \\
\hline df & 890 & \\
\hline t Stat & 3.63 & \\
\hline $\mathrm{P}(\mathrm{T}<=\mathrm{t})$ one-tail & 1.47E-04 & \\
\hline t Critical one-tail & 1.65 & \\
\hline$P(T<=t)$ two-tail & 2.94E-04 & \\
\hline \multirow[t]{2}{*}{ t Critical two-tail } & 1.96 & \\
\hline & $5^{\text {th }}$ Injection & $4^{\text {th }}$ Injection \\
\hline Mean & 98.7 & 92.2 \\
\hline Variance & 1034 & 933 \\
\hline Observations & 216 & 502 \\
\hline Hypothesized Mean Difference & 0 & \\
\hline df & 389 & \\
\hline t Stat & 2.54 & \\
\hline $\mathrm{P}(\mathrm{T}<=\mathrm{t})$ one-tail & $5.8 \mathrm{E}-03$ & \\
\hline t Critical one-tail & 1.65 & \\
\hline$P(T<=t)$ two-tail & $1.2 \mathrm{E}-02$ & \\
\hline t Critical two-tail & 1.96 & \\
\hline
\end{tabular}

(b) TCNM

\begin{tabular}{|c|c|c|}
\hline & $1^{\text {st }}$ Injection & Before \\
\hline Mean & 74.2 & 63.7 \\
\hline Variance & 537 & 526 \\
\hline Observations & 511 & 663 \\
\hline Hypothesized Mean Difference & 0 & \\
\hline Df & 1092 & \\
\hline t Stat & 7.75 & \\
\hline $\mathrm{P}(\mathrm{T}<=\mathrm{t})$ one-tail & $1.07 \mathrm{E}-14$ & \\
\hline t Critical one-tail & 1.65 & \\
\hline $\mathrm{P}(\mathrm{T}<=\mathrm{t})$ two-tail & $2.14 \mathrm{E}-14$ & \\
\hline \multirow[t]{2}{*}{ t Critical two-tail } & 1.96 & \\
\hline & $2^{\text {nd }}$ Injection & jection \\
\hline Mean & 79.1 & 74.2 \\
\hline Variance & 534 & 537 \\
\hline Observations & 509 & 511 \\
\hline Hypothesized Mean Difference & 0 & \\
\hline Df & 1018 & \\
\hline t Stat & 3.31 & \\
\hline
\end{tabular}


$P(T<=t)$ one-tail

4.8E-04

t Critical one-tail

1.65

$\mathrm{P}(\mathrm{T}<=\mathrm{t})$ two-tail

9.7E-04

t Critical two-tail

1.96

\begin{tabular}{|c|c|c|}
\hline & $3^{\text {rd }}$ Injection & $2^{\text {nd }}$ Injection \\
\hline Mean & 81.9 & 79.1 \\
\hline Variance & 800 & 534 \\
\hline Observations & 501 & 509 \\
\hline Hypothesized Mean Difference & 0 & \\
\hline df & 964 & \\
\hline t Stat & 1.73 & \\
\hline$P(T<=t)$ one-tail & $4.2 \mathrm{E}-2$ & \\
\hline t Critical one-tail & 1.65 & \\
\hline $\mathrm{P}(\mathrm{T}<=\mathrm{t})$ two-tail & 8.4E-02 & \\
\hline \multirow[t]{2}{*}{ t Critical two-tail } & 1.96 & \\
\hline & $4^{\text {th }}$ Injection & $3^{\text {rd }}$ Injection \\
\hline Mean & 86.5 & 81.9 \\
\hline Variance & 863 & 800 \\
\hline Observations & 502 & 501 \\
\hline Hypothesized Mean Difference & 0 & \\
\hline df & 1000 & \\
\hline t Stat & 2.53 & \\
\hline$P(T<=t)$ one-tail & 5.9E-03 & \\
\hline t Critical one-tail & 1.65 & \\
\hline$P(T<=t)$ two-tail & $1.2 \mathrm{E}-02$ & \\
\hline \multirow[t]{2}{*}{ t Critical two-tail } & 1.96 & \\
\hline & $5^{\text {th }}$ Injection & $4^{\text {th }}$ Injection \\
\hline Mean & 95.8 & 86.5 \\
\hline Variance & 842 & 864 \\
\hline Observations & 319 & 502 \\
\hline Hypothesized Mean Difference & 0 & \\
\hline df & 683 & \\
\hline t Stat & 4.47 & \\
\hline$P(T<=t)$ one-tail & 4.5E-06 & \\
\hline t Critical one-tail & 1.65 & \\
\hline$P(T<=t)$ two-tail & 9E-06 & \\
\hline t Critical two-tail & 1.96 & \\
\hline
\end{tabular}


2. Width

(a) 4-Cl-NB

\begin{tabular}{lrr}
\hline & $5^{\text {th }}$ Injection & Before \\
\hline Mean & 8.42 & 8.96 \\
Variance & 10.9 & 15.9 \\
Observations & 216 & 663 \\
Hypothesized Mean Difference & 0 & \\
df & 562 & \\
t Stat & -1.96 & \\
P $(T<=t)$ one-tail & $5.06 \mathrm{E}-06$ & \\
t Critical one-tail & 1.65 & \\
$\mathrm{P}(\mathrm{T}<=\mathrm{t})$ two-tail & $1.01 \mathrm{E}-05$ & \\
t Critical two-tail & 1.96 & \\
\hline
\end{tabular}

(b) TCNM

\begin{tabular}{lrr}
\hline & $5^{\text {th }}$ Injection & Before \\
\hline Mean & 8.52 & 8.96 \\
Variance & 9.23 & 15.9 \\
Observations & 319 & 663 \\
Hypothesized Mean Difference & 0 & \\
df & 896 & \\
t Stat & -1.93 & \\
P $(T<=t)$ one-tail & $1.2 \mathrm{E}-03$ & \\
t Critical one-tail & 1.65 & \\
$\mathrm{P}(T<=t)$ two-tail & $2.3 \mathrm{E}-02$ & \\
t Critical two-tail & 1.96 & \\
\hline
\end{tabular}




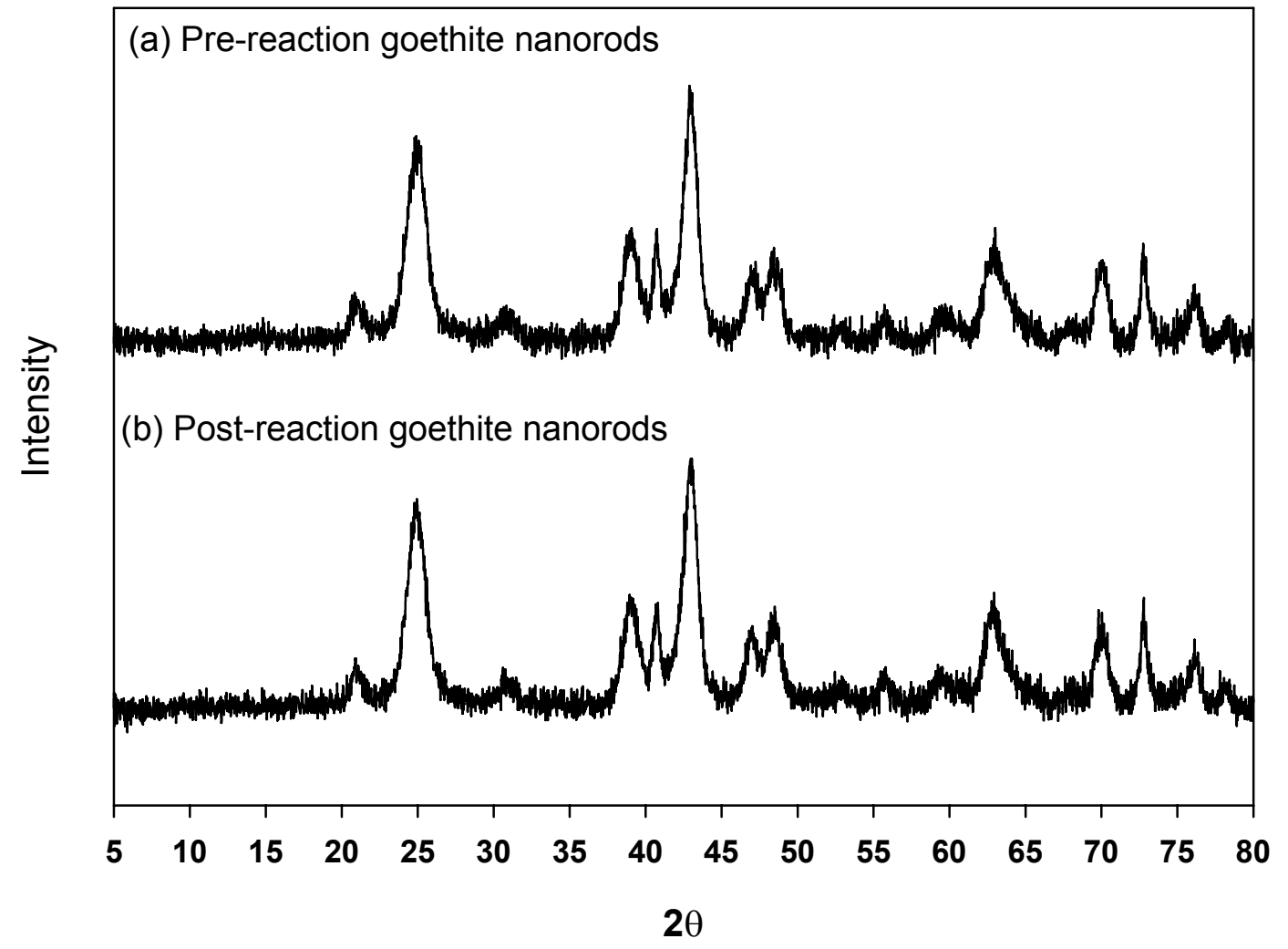

Figure S4. X-ray diffraction patterns of (a) pre- and (b) post-reaction goethite 


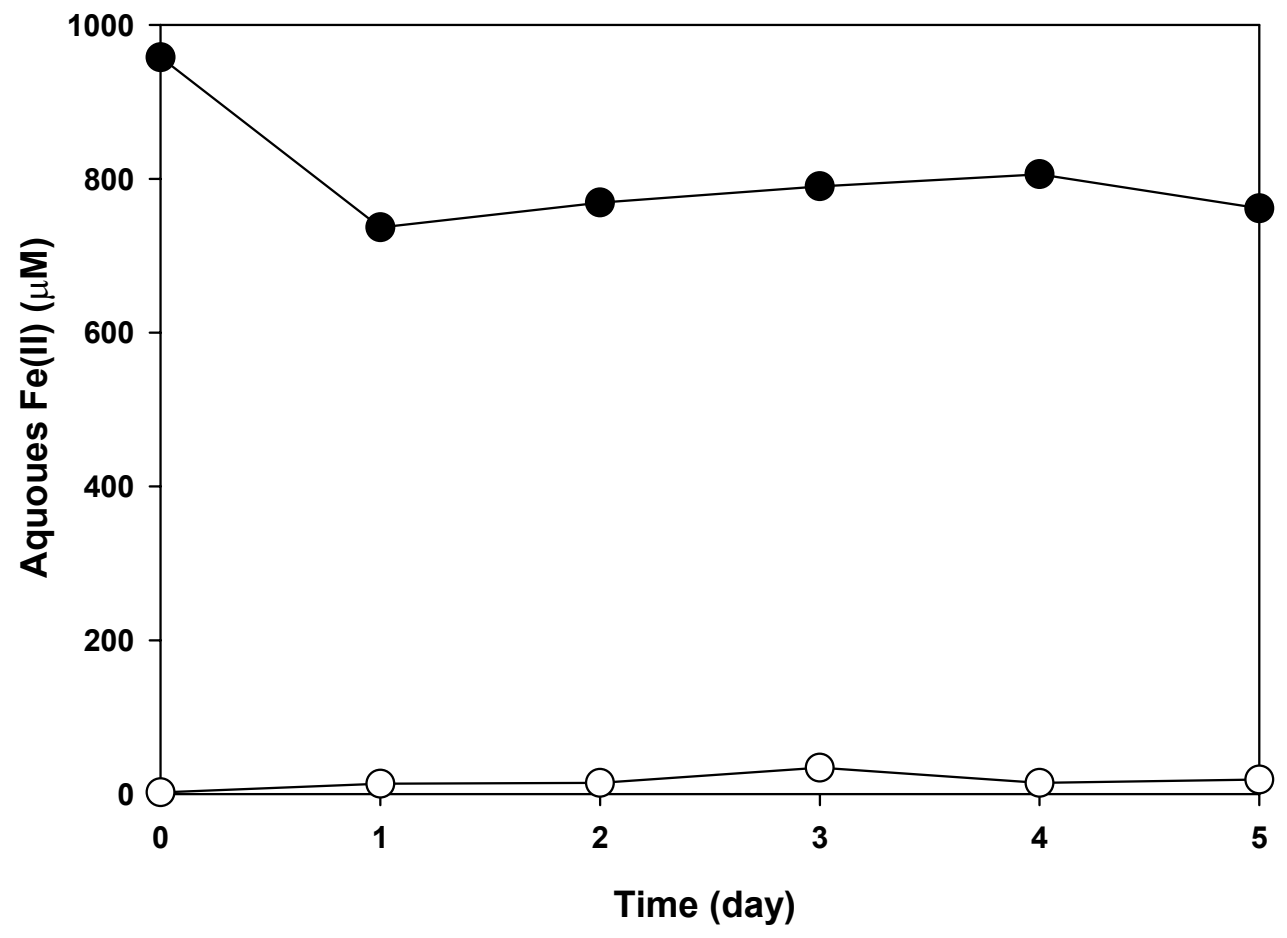

Figure S5.Aqueous Fe(II) concentration in control experiments: $0.65 \mathrm{~g} / \mathrm{L}$ goethite only $(O)$ and $1 \mathrm{mM} \mathrm{Fe}(\mathrm{II})+0.65 \mathrm{~g} / \mathrm{L}$ goethite (@). Fe(II) was injected in the reactor at Day 0 and the drop of $\mathrm{Fe}(\mathrm{II})$ concentration at Day 1 is due to $\mathrm{Fe}(\mathrm{II})$ adsorption onto goethite. 
Section S4. Calculation of the predicted lengths of new goethite via mass balance

Assumption: The length of goethite particle increased due to reaction with organic contaminants

Step1. Moles of organic compound reduced (A)

Step2. Moles of electrons used for the reduction $(B)=6 \times A$

Step3. Mass of newly formed goethite $(\mathrm{C})=\mathrm{B} \times$ Molecular weight of goethite

Step4. Ratio of new goethite to total mass $(\mathrm{R})=(\mathrm{C}+\mathrm{X}) / \mathrm{X}$

Step5. Predicted length $(\mathrm{L} 2)=\mathrm{R} \times \mathrm{L} 1$

$\mathrm{X}$ : Mass of goethite in previous injection

L1: Average length of goethite in previous injection

$\mathrm{L}_{\text {actual }}$ : Mean value of goethite length from particle size analysis

\begin{tabular}{c|c|c|c|c|c|c|c|c}
\hline Compound & Injection & $\begin{array}{c}\mathrm{A} \\
(\mu \mathrm{moles})\end{array}$ & $\begin{array}{c}\mathrm{B} \\
(\mu \mathrm{moles})\end{array}$ & $\begin{array}{c}\mathrm{C} \\
(\mathrm{mg})\end{array}$ & $\mathrm{R}$ & $\mathrm{L} 1$ & $\mathrm{~L} 2$ & $\mathrm{~L}_{\text {actual }}$ \\
\hline \multirow{5}{*}{$4-\mathrm{Cl}-\mathrm{NB}$} & $1^{\text {st }}$ & 12.67 & 76.01 & 6.75 & 1.084 & 63.73 & 69.11 & 71.15 \\
\cline { 2 - 9 } & $2^{\text {nd }}$ & 11.69 & 70.11 & 6.23 & 1.072 & 71.15 & 76.26 & 75.29 \\
\cline { 2 - 9 } & $3^{\text {rd }}$ & 12.55 & 75.28 & 6.69 & 1.077 & 75.29 & 81.13 & 85.13 \\
\cline { 2 - 9 } & $4^{\text {th }}$ & 11.81 & 70.85 & 6.29 & 1.072 & 85.13 & 91.31 & 92.16 \\
\cline { 2 - 9 } & $5^{\text {th }}$ & 11.94 & 71.59 & 6.36 & 1.073 & 92.16 & 98.95 & 98.70 \\
\hline \multirow{5}{*}{ TCNM } & $1^{\text {st }}$ & 12.55 & 75.28 & 6.69 & 1.083 & 63.73 & 69.06 & 74.26 \\
\cline { 2 - 9 } & $2^{\text {nd }}$ & 11.93 & 71.59 & 6.36 & 1.073 & 74.26 & 79.71 & 79.05 \\
\cline { 2 - 9 } & $3^{\text {rd }}$ & 11.81 & 70.85 & 6.29 & 1.073 & 79.05 & 84.81 & 81.86 \\
\cline { 2 - 9 } & $4^{\text {th }}$ & 12.42 & 74.54 & 6.62 & 1.077 & 81.86 & 88.14 & 86.49 \\
\cline { 2 - 8 } & $5^{\text {th }}$ & 12.55 & 75.28 & 6.69 & 1.077 & 86.49 & 93.17 & 92.47 \\
\hline
\end{tabular}

Section S5. Visual MINTEQ input and output

Input

\begin{tabular}{|l|c|c|}
\hline \hline Components & Total Conc. $(\mathrm{mM})$ & Remarks \\
\hline $\mathrm{H}^{+}$ & $1.00 \mathrm{E}-07$ & Fixed \\
\hline $\mathrm{Cl}^{-1}$ & 2 & \\
\hline $\mathrm{Fe}^{2+}$ & 1 & \\
\hline
\end{tabular}

Output

\begin{tabular}{|l|c|c|c|}
\hline \hline & Conc. (M) & Activity & Log activity \\
\hline $\mathrm{Cl}^{-1}$ & $2.00 \mathrm{E}-03$ & $1.88 \mathrm{E}-03$ & $-2.73 \mathrm{E}+00$ \\
\hline $\mathrm{Fe}(\mathrm{OH})_{2(\mathrm{aq})}$ & $1.54 \mathrm{E}-10$ & $1.54 \mathrm{E}-10$ & $-9.81 \mathrm{E}+00$ \\
\hline $\mathrm{Fe}(\mathrm{OH})_{3}^{-}$ & $5.06 \mathrm{E}-14$ & $4.77 \mathrm{E}-14$ & $-1.33 \mathrm{E}+01$ \\
\hline $\mathrm{Fe}$ & $9.96 \mathrm{E}-04$ & $7.85 \mathrm{E}-04$ & $-3.11 \mathrm{E}+00$ \\
\hline $\mathrm{FeCl}^{+2}$ & $9.90 \mathrm{E}-07$ & $9.33 \mathrm{E}-07$ & $-6.03 \mathrm{E}+00$ \\
\hline $\mathrm{FeOH}^{+}$ & $2.66 \mathrm{E}-06$ & $2.50 \mathrm{E}-06$ & $-5.60 \mathrm{E}+00$ \\
\hline $\mathrm{H}^{+1}$ & $1.06 \mathrm{E}-07$ & $1.00 \mathrm{E}-07$ & $-7.00 \mathrm{E}+00$ \\
\hline $\mathrm{OH}^{-}$ & $8.50 \mathrm{E}-08$ & $8.01 \mathrm{E}-08$ & $-7.10 \mathrm{E}+00$ \\
\hline
\end{tabular}




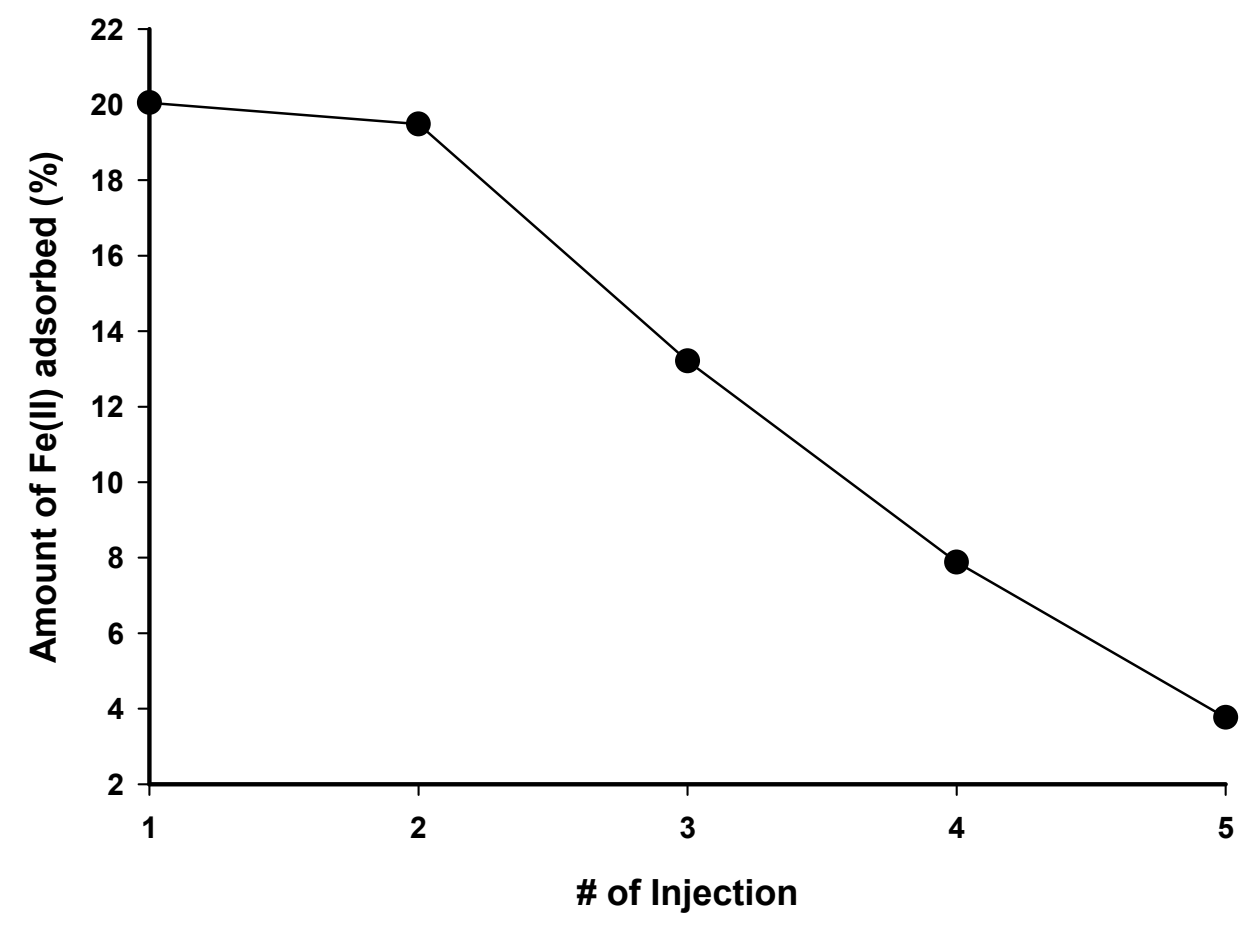

Figure S6. Initial percentage of total Fe(II) adsorbed in the sequential spike experiment of 4-ClNB (cf. Figure 1a)

Table S2. Surface area of goethite nanoparticles after sequential spike experiments

\begin{tabular}{|c|c|c|c|c|c|c|}
\hline \multirow{2}{*}{$\begin{array}{l}\text { Number of } \\
\text { Injection }\end{array}$} & \multicolumn{3}{|c|}{ 4-Cl-NB } & \multicolumn{3}{|c|}{ TCNM } \\
\hline & $\begin{array}{l}\mathrm{SA}_{\text {Total }} \\
\left(\mathrm{m}^{2} / \mathrm{g}\right)\end{array}$ & $\begin{array}{c}\mathrm{SA}_{021} / \mathrm{SA}_{\text {Total }} \\
(\%)\end{array}$ & $\begin{array}{c}\mathrm{SA}_{110} / \mathrm{SA}_{\text {Total }} \\
(\%)\end{array}$ & $\begin{array}{l}\mathrm{SA}_{\text {Total }} \\
\left(\mathrm{m}^{2} / \mathrm{g}\right)\end{array}$ & $\begin{array}{c}\mathrm{SA}_{021} / \mathrm{SA}_{\text {Total }} \\
(\%)\end{array}$ & $\begin{array}{c}\mathrm{SA}_{110} / \mathrm{SA}_{\text {Total }} \\
(\%)\end{array}$ \\
\hline Before & $218.6 \pm 86.4$ & 4.5 & 95.5 & $218.6 \pm 86.4$ & 4.5 & 95.5 \\
\hline $1^{\text {st }}$ & $245.6 \pm 92.2$ & 3.9 & 96.1 & $258.4 \pm 70.3$ & 2.7 & 97.3 \\
\hline $2^{\text {nd }}$ & $272.6 \pm 84.4$ & 2.7 & 97.3 & $245.9 \pm 64.5$ & 2.7 & 97.3 \\
\hline $3^{\text {rd }}$ & $238.8 \pm 70.5$ & 2.7 & 97.3 & $260.1 \pm 74.7$ & 2.5 & 97.5 \\
\hline $4^{\text {th }}$ & $231.8 \pm 68.2$ & 2.5 & 97.5 & $250.5 \pm 68.5$ & 2.4 & 95.6 \\
\hline $5^{\text {th }}$ & $218.7 \pm 73.7$ & 2.6 & 97.4 & $213.3 \pm 70.7$ & 2.7 & 95.3 \\
\hline
\end{tabular}

$\mathrm{SA}_{\text {Total }}$ is surface area calculated using TEM measurement.

$\mathrm{SA}_{021}$ is surface area of $(021)$ faces

$\mathrm{SA}_{110}$ is surface area of (110) faces 